\title{
Does Extended Use of Clopidogrel-Based Dual Anti-Platelet Therapy Increase the Risk of Gastrointestinal Bleeding?
}

\author{
Hafsa Abbas ${ }^{a}$, c, Suresh Kumar Nayudua, Madhavi Ravia, Muhammad Saad ${ }^{\mathrm{b}}$, Kashyap Bathini ${ }^{\mathrm{b}}$, \\ Pranav Ravi ${ }^{\mathrm{b}}$, Swathi Roy ${ }^{\mathrm{b}}$, Divya Arya ${ }^{\mathrm{b}}$, Sridhar Chilimuri ${ }^{\mathrm{b}}$
}

\begin{abstract}
Background: Clopidogrel-based dual anti-platelet therapy (CDAPT) has shown significant benefits in the management of coronary artery disease (CAD), up to 1-year duration. Gastrointestinal bleeding (GIB) is one of the limiting factors for prolonged use of CDAPT.

Methods: We identified all patients taking CDAPT from our ambulatory clinics. Demographic, clinical, laboratory and pharmacological data were abstracted. American Heart Association (AHA) guidelines were used to determine the duration of CDAPT therapy. The study population was divided into two groups based on the duration of therapy. Individuals who received CDAPT more than 12 months were deemed as extended use.
\end{abstract}

Results: A total of 351 patients with CAD were taking CDAPT. Majority of patients $(276 / 351,79 \%)$ were taking CDAPT beyond 1 year. There were no differences in baseline characteristics between the two groups. There was no significant difference in the incidence of GIB between the two groups. However, in subgroup analysis, there was a significant difference in the incidence of GIB in men. Men who were taking CDAPT beyond 12 months had almost three times higher incidence of GIB compared to those who were taking less than 12 months $(25 \%$ vs. $8 \%, \mathrm{P}=0.04)$. The excess GIB in men prevailed despite adjusting for non-steroidal anti-inflammatory drugs (NSAIDs) or direct oral anticoagulant (DOAC) use.

Conclusions: We found that a majority of patients were taking CDAPT beyond the recommended duration. We observed that men taking CDAPT for an extended duration had a three times higher incidence of GIB. It would be reasonable for physicians to be aware of the higher risk of GIB in men and carefully assess the risks and benefits of extended use of CDAPT.

Keywords: Gastrointestinal bleeding; Dual anti-platelet therapy;

Manuscript submitted April 6, 2020, accepted May 20, 2020

Published online August 14, 2020

aDepartment of Medicine, Division of Gastroenterology, Bronxcare Health System, Bronx, NY 10457, USA

bDepartment of Medicine, Bronxcare Health System, Bronx, NY 10457, USA ${ }^{c}$ Corresponding Author: Hafsa Abbas, Department of Medicine, Division of Gastroenterology, Bronxcare Health System, Bronx, NY 10457, USA.

Email: hafsaabbas20@gmail.com
Clopidogrel; Coronary artery disease; Extended use

\section{Introduction}

Clopidogrel-based dual antiplatelet therapy (CDAPT) comprising of aspirin and a P2Y12 inhibitor (clopidogrel) has been recommended by American College of Cardiology/American Heart Association (ACC/AHA) for patients with coronary artery disease $(\mathrm{CAD})$ presenting with acute coronary syndrome (ACS). The recommended duration of CDAPT use is at least 12 months in patients with or without revascularization (percutaneous coronary intervention (PCI), coronary artery bypass grafting $(\mathrm{CABG}))[1-10]$. The recommendations regarding the extended use of CDAPT beyond 1 year are not well-documented. The decision regarding continued use beyond the recommended period varies from individual to individual, based on risks versus benefits and at the discretion of the cardiologist.

Multiple studies have shown the benefits of CDAPT therapy beyond 1 year after coronary stenting in reducing the major cardiovascular and cerebrovascular events as well as stent thrombosis [11]. Additionally, the prolonged use of CDAPT in these patients also elevates the risk of bleeding (including intracranial hemorrhage and gastrointestinal hemorrhage) and related adverse outcomes [11, 12].

Gastrointestinal bleeding (GIB) is a significant complication associated with CDAPT use in patients with CAD [13] that has been well established by multiple randomized controlled trials [1, 14-16]. A significant episode of GIB in such patients might pose a clinical dilemma for the physicians prompting the discontinuation of the CDAPT risking the occurrence of ischemic events. However, the continued use of CDAPT for an extended period in patients with stable ischemic heart disease exposes them to the development of adverse events such as GIB which can be fatal in certain cases [17].

With our study, we aim to evaluate the risks of significant GIB in association with extended use of CDAPT, and the need to carefully review the indications of the use beyond the recommended period to avoid dreadful complications.

\section{Materials and Methods}

Institutional Review Board Approval was obtained for this 
Table 1. Comparison of the Demographic Data of the Two Groups

\begin{tabular}{llll}
\hline Demographic feature & Standard use of CDAPT $(\mathbf{n}=\mathbf{7 5})$ & Extended use of CDAPT $(\mathbf{n}=\mathbf{2 7 6})$ & P value \\
\hline Gender & & & $140(51 \%)$ \\
$\quad$ Male & $37(49 \%)$ & $136(49 \%)$ & 0.79 \\
$\quad$ Female & $38(51 \%)$ & 65 & 0.79 \\
Mean age & 63 & $13(5 \%)$ & 0.16 \\
Use of NSAID/DOAC/VKA & $1(1.5 \%)$ & 0.2 \\
\hline
\end{tabular}

NSAID: non-steroidal anti-inflammatory drug; DOAC: direct oral anticoagulant; VKA: vitamin K antagonists.

Table 2. Incidence of GIB

\begin{tabular}{llll} 
& GIB in extended use of dual- & GIB in recommended use of & P value \\
antiplatelet therapy & dual-antiplatelet therapy & 0.26 \\
\hline Total population (\%) & 22 & 16 & 0.04 \\
Male (\%) & 25 & 8 & 0.65 \\
\hline Female (\%) & 20 & 24 & 0 \\
\hline
\end{tabular}

GIB: gastrointestinal bleeding.

study, and it was conducted in compliance with the ethical standards of the responsible institution on human subjects as well as with the Helsinki Declaration and institutional ethical guidelines for the care.

We conducted a retrospective observational study at our institution between January 1, 2015 and December 31, 2015. We identified all patients taking CDAPT from the electronic medical records (EMRs) at our ambulatory clinics during this period. The demographic, clinical, laboratory and pharmacological data were abstracted from the EMRs. Patients who had developed GIB while on CDAPT were identified using the International Classification of Diseases (ICD)-9/10 codes. We used ACC/AHA guidelines to determine the duration of therapy indicated for each patient.

The study population was divided into two main groups based on the duration of therapy: recommended (CDAPT use for 1 year) versus extended group (patients who received CDAPT for more than 12 months were deemed as extended use). Non-steroidal anti-inflammatory drugs (NSAIDs) or di- rect oral anticoagulant (DOAC) use was also recorded. We compared the incidence of GIB episodes between these two groups. Subgroup analysis within each group was also performed and the results were compared. The data were analyzed using standard statistical methods and JMP 13 software.

\section{Results}

A total of 351 patients with CAD were confirmed to be taking CDAPT. Seventy-five out of 351(21\%) were noted to have been taking the therapy for the recommended duration, while the majority of the patients (i.e. 276 out of $351,79 \%$ ) were taking CDAPT beyond 1 year without a clear indication. There were no differences in baseline characteristics between the two groups (Table 1). We found no significant difference in the incidence of GIB between these two groups. The incidence of GIB in the recommended duration group was $16 \%$ versus $22 \%$ in the extended use group $(\mathrm{P}=0.26)$.

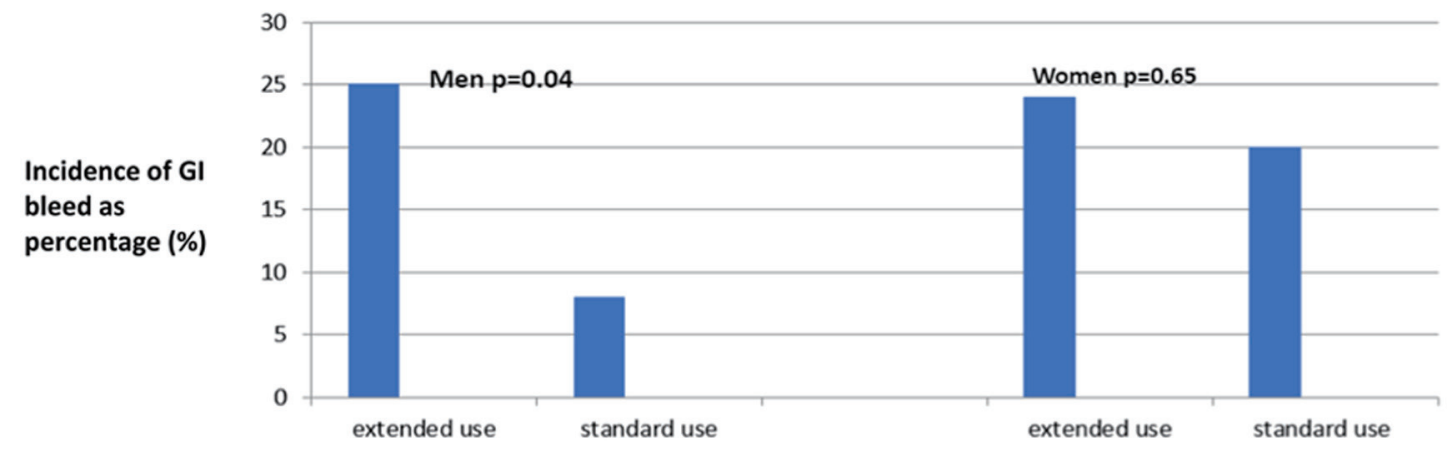

Figure 1. Incidence of GIB in the subgroups. GIB: gastrointestinal bleeding. 
Interestingly, in subgroup analysis, there was a significant difference in the incidence of GIB in men (Table 2, Fig. 1), who were taking CDAPT beyond 12 months. We noticed a three times higher incidence of GIB in men taking CDAPT for prolonged time compared to those who were taking CDAPT for a recommended period of 12 months $(25 \%$ vs. $8 \%, \mathrm{P}=$ 0.04). However, there was no difference in the incidence of GIB in women irrespective of the duration of the CDAPT treatment $(20 \%$ vs. $24 \%, \mathrm{P}=0.65)$.

\section{Discussion}

Addition of antiplatelets to aspirin has been shown to significantly increase the risk of GIB as compared to aspirin alone $[18,19]$. Multiple studies have confirmed the increased incidence of GIB in patients receiving aspirin and other antiplatelet agents for myocardial infarction (MI)/ACS, the use of CDAPT being the most important predictor $[3,15,16]$. The Valsartan in Acute Myocardial Infarction (VALIANT) trial studied multiple risk factors associated with increased GIB in patient post MI. Among other risk factors such as older age, comorbidities, alcohol use, non-white race, anticoagulant use, renal disease, male gender (similar to our study), the use of DAPT was the most important predictor of GIB [16]. The extended use of DAPT beyond 1 year after placement of drug eluting stent (DES) has also been shown to decrease ischemic events; however, it was associated with increased risk of bleeding [11]. As per literature review, the GIB in patients on CDAPT is mostly from upper GI tract and there is not enough data on the protective role of prophylactic use of proton pump inhibitor (PPI) [18, 20].

In our study we found that a majority of patients were taking CDAPT beyond the recommended duration. This is not consistent with the current guidelines and may vary from case to case based on the risk factors and the physicians' decision weighing the risks versus benefits. We found that even though there was no difference in GIB between the two groups, men taking CDAPT for an extended duration had a three times higher incidence of GIB as compared to their female counterparts. This differential risk of bleeding based on gender is unclear but is similar to a previous study that found male gender to be a risk factor for GIB in patients on DAPT [16].

We understand the limitations of our study and the need for further studies to identify the unique risk of GIB in men taking CDAPT for an extended duration beyond the usual recommended time. However, it would be reasonable for physicians to be aware of the higher risk of GIB in men and carefully assess the risks and benefits of extended use of CDAPT particularly in high risk patients.

\section{Conclusions}

We conclude that men taking CDAPT for CAD and ACS are at higher risk for GIB than women. Further studies would be needed to identify the unique risk of GIB in men taking
CDAPT. However, cautious use of extended duration use of CDAPT could improve outcomes.

\section{Acknowledgments}

On behalf of all authors, I acknowledge the findings in the research manuscript.

\section{Financial Disclosure}

None to declare.

\section{Conflict of Interest}

None to declare.

\section{Informed Consent}

Not applicable.

\section{Author Contributions}

SC, HA, and SKN were responsible for conceptualization and IRB; HA, MS, MR, KB, PR, SR and DA contributed to data collection; SKN contributed to data analysis; HA, SKN and SC were involved in manuscript writing editing and proofreading.

\section{Data Availability}

The authors declare that data supporting the findings of this study are available within the article.

\section{References}

1. Steinhubl SR, Berger PB, Mann JT, 3rd, Fry ET, DeLago A, Wilmer C, Topol EJ, et al. Early and sustained dual oral antiplatelet therapy following percutaneous coronary intervention: a randomized controlled trial. JAMA. 2002;288(19):2411-2420.

2. Mehta SR, Yusuf S, Peters RJ, Bertrand ME, Lewis BS, Natarajan MK, Malmberg K, et al. Effects of pretreatment with clopidogrel and aspirin followed by long-term therapy in patients undergoing percutaneous coronary intervention: the PCI-CURE study. Lancet. 2001;358(9281):527533.

3. Yusuf S, Zhao F, Mehta SR, Chrolavicius S, Tognoni G, Fox KK, Clopidogrel in Unstable Angina to Prevent Recurrent Events Trial Investigators. Effects of clopidogrel in addition to aspirin in patients with acute coronary syndromes without ST-segment elevation. N Engl J Med. 2001;345(7):494-502. 
4. Wallentin L, Becker RC, Budaj A, Cannon CP, Emanuelsson H, Held C, Horrow J, et al. Ticagrelor versus clopidogrel in patients with acute coronary syndromes. N Engl J Med. 2009;361(11):1045-1057.

5. Wiviott SD, Braunwald E, McCabe CH, Montalescot G, Ruzyllo W, Gottlieb S, Neumann FJ, et al. Prasugrel versus clopidogrel in patients with acute coronary syndromes. N Engl J Med. 2007;357(20):2001-2015.

6. Montalescot G, Wiviott SD, Braunwald E, Murphy SA, Gibson CM, McCabe CH, Antman EM, et al. Prasugrel compared with clopidogrel in patients undergoing percutaneous coronary intervention for ST-elevation myocardial infarction (TRITON-TIMI 38): double-blind, randomised controlled trial. Lancet. 2009;373(9665):723-731.

7. Steg PG, James S, Harrington RA, Ardissino D, Becker $\mathrm{RC}$, Cannon CP, Emanuelsson H, et al. Ticagrelor versus clopidogrel in patients with ST-elevation acute coronary syndromes intended for reperfusion with primary percutaneous coronary intervention: A Platelet Inhibition and Patient Outcomes (PLATO) trial subgroup analysis. Circulation. 2010;122(21):2131-2141.

8. Brar SS, Kim J, Brar SK, Zadegan R, Ree M, Liu IL, Mansukhani P, et al. Long-term outcomes by clopidogrel duration and stent type in a diabetic population with de novo coronary artery lesions. J Am Coll Cardiol. 2008;51(23):2220-2227.

9. Eisenstein EL, Anstrom KJ, Kong DF, Shaw LK, Tuttle RH, Mark DB, Kramer JM, et al. Clopidogrel use and long-term clinical outcomes after drug-eluting stent implantation. JAMA. 2007;297(2):159-168.

10. Sabatine MS, Cannon CP, Gibson CM, Lopez-Sendon JL, Montalescot G, Theroux P, Lewis BS, et al. Effect of clopidogrel pretreatment before percutaneous coronary intervention in patients with ST-elevation myocardial infarction treated with fibrinolytics: the PCI-CLARITY study. JAMA. 2005;294(10):1224-1232.

11. Mauri L, Kereiakes DJ, Yeh RW, Driscoll-Shempp P, Cutlip DE, Steg PG, Normand SL, et al. Twelve or 30 months of dual antiplatelet therapy after drug-eluting stents. N Engl J Med. 2014;371(23):2155-2166.

12. Lippi G, Franchini M, Cervellin G. Diagnosis and management of ischemic heart disease. Semin Thromb Hemost. 2013;39(2):202-213.
13. Chandrasekhar J, Bansilal S, Baber U, Sartori S, Aquino M, Farhan S, Vogel B, et al. Impact of proton pump inhibitors and dual antiplatelet therapy cessation on outcomes following percutaneous coronary intervention: Results From the PARIS Registry. Catheter Cardiovasc Interv. 2017;89(7):E217-E225.

14. Peters RJ, Mehta SR, Fox KA, Zhao F, Lewis BS, Kopecky SL, Diaz R, et al. Effects of aspirin dose when used alone or in combination with clopidogrel in patients with acute coronary syndromes: observations from the Clopidogrel in Unstable angina to prevent Recurrent Events (CURE) study. Circulation. 2003;108(14):1682-1687.

15. Diener HC, Bogousslavsky J, Brass LM, Cimminiello C, Csiba L, Kaste M, Leys D, et al. Aspirin and clopidogrel compared with clopidogrel alone after recent ischaemic stroke or transient ischaemic attack in high-risk patients (MATCH): randomised, double-blind, placebo-controlled trial. Lancet. 2004;364(9431):331-337.

16. Moukarbel GV, Signorovitch JE, Pfeffer MA, McMurray $\mathrm{JJ}$, White $\mathrm{HD}$, Maggioni $\mathrm{AP}$, Velazquez EJ, et al. Gastrointestinal bleeding in high risk survivors of myocardial infarction: the VALIANT Trial. Eur Heart J. 2009;30(18):2226-2232.

17. Nikolsky E, Mehran R, Stone GW. Gastrointestinal bleeding in percutaneous coronary intervention and acute coronary syndromes. Am J Cardiol. 2009;104(5 Suppl):22C29C.

18. Hallas J, Dall M, Andries A, Andersen BS, Aalykke C, Hansen JM, Andersen M, et al. Use of single and combined antithrombotic therapy and risk of serious upper gastrointestinal bleeding: population based case-control study. BMJ. 2006;333(7571):726.

19. Aronow HD, Steinhubl SR, Brennan DM, Berger PB, Topol EJ, Investigators C. Bleeding risk associated with 1 year of dual antiplatelet therapy after percutaneous coronary intervention: Insights from the Clopidogrel for the Reduction of Events During Observation (CREDO) trial. Am Heart J. 2009;157(2):369-374.

20. Liberopoulos EN, Elisaf MS, Tselepis AD, Archimandritis A, Kiskinis D, Cokkinos D, Mikhailidis DP. Upper gastrointestinal haemorrhage complicating antiplatelet treatment with aspirin and/or clopidogrel: where we are now? Platelets. 2006;17(1):1-6. 